\title{
Proximate Versus Ultimate Causation and Evo-Devo
}

Rachael L. Brown

School of Philosophy, Research School of Social Sciences, The Australian National University, Canberra, ACT, Australia

Email: rachael.brown@anu.edu.au

\begin{abstract}
Made famous by Ernst Mayr (1961), the distinction between proximate and ultimate causation in biological explanation is widely seen as a key tenet of evolutionary theory and a central organizing principle for evolutionary research. The study of immediate, individual-level mechanistic causes of development or physiology ("proximate causation") is distinguished from the study of historical, population-level statistical causes in evolutionary biology ("ultimate causation"). Since evolutionary developmental biology (evo-devo) is a field that explicitly uses so-called "proximate" sciences such as developmental biology, morphology, and embryology in the study of evolution, it challenges the standard construal of the proximateultimate distinction and its associated account of causation. The exact nature of the challenge and its ramifications for the viability of the distinction more broadly are contested, but these conceptual questions are central to the status and significance of evo-devo in contemporary evolutionary biology.
\end{abstract}

Keywords: Proximate causation - Ultimate causation - Mayr

\section{Introduction}

The distinction between "proximate" causes and "ultimate" causes and corresponding differences in biological explanation are commonly invoked when delineating different domains of inquiry within the life sciences. According to this prevalent approach, made famous by Ernst Mayr (1961), explaining any particular trait or its features within an organism involves invoking one of two types of cause - proximate or ultimate. Roughly, proximate causes are individual-level, mechanistic phenomena, while ultimate causes are population-level, evolutionary phenomena. Mayr uses the annual southward migration of warblers to illustrate his point. One possible explanation for the migration of warblers from North America south for the winter references its "proximate" causes. These include, for example, the interaction between the physiology of particular warblers and changes in the environment in the northern hemisphere during autumn that trigger their migration behavior, such as reducing temperature and light levels. Alternatively, the migration could be accounted for by reference to its "ultimate" causes. These include the historical lack of food in the northern hemisphere and its selective impact upon the genetic makeup of ancestral warbler populations over time. These two types of causes, in turn, are said to delineate independent research domains within biology - proximate causation is the focus of fields such as developmental biology, physiology, and anatomy, whereas ultimate causation is the focus of evolutionary biology, especially areas such as population genetics and behavioral ecology.

The proximate-ultimate dichotomy has played a key role in shaping the landscape of biology and evolutionary biology in particular, for the past half century, which is observable in university infrastructure where ecology and evolutionary biology are often separate administrative units from genetics, cell biology, and development.

Evo-devo is a research field that takes seriously the potential for developmental processes within individuals (apparent proximate causes) to play an important role in evolution at the 
population level (the domain of ultimate causes). This challenges the premise that proximate and ultimate causes are distinct and that inquiry into them is independent from each other. Therefore, understanding the motivation behind evo-devo as a field involves understanding why one might step outside the research framework encapsulated by this distinction. This chapter considers the challenge that evo-devo presents to this traditional conception of causation and its associated division of cognitive labor within biology. Alternative conceptions of causation and explanation found in evo-devo research help to situate its agenda within evolutionary biology, clarify its motivations, and account for the organization of inquiry in this field. To appreciate the specific differences of these alternatives, a more detailed overview of Mayr's distinction, its origins, and roles in contemporary evolutionary biology is needed.

\section{The Dichotomy: Proximate and Ultimate Causation Explained}

The historical context in which the idea that there are two, complementary, ways of accounting for the features of organisms - proximate causes and ultimate causes - came to prominence is perhaps as important as the details of the distinction itself for understanding its relationship to evo-devo. The idea is most commonly associated with Mayr's 1961 Science article "Cause and Effect in Biology" although his thinking on the matter goes right back to his $\mathrm{PhD}$ thesis and other work on bird migration in the late 1920s (Beatty 1994). The 1961 article came at a time when evolutionary biology was under threat; in the late 1950s and 1960s, following Watson and Crick's discovery of DNA, the central place of evolutionary biology was under threat from the boom in molecular biology and genetics. It is only through this historical lens that Mayr's intent in the 1961 article is really clear; it is an attempt to stamp out a clear domain for evolutionary biology (i.e., the study of ultimate causes).

According to Mayr ( 1961), proximate causes "govern the responses of an individual (and his organs) to immediate factors of the environment" (p. 1503). These causes are mechanistic in nature and account for how the individual organisms in question produce the trait of interest or manifest a particular feature. For Mayr, proximate causes offer answers to "how" questions, rather than "why" questions. For example, biologists interested in the single-toed hooves seen in zebras, horses, and other equines might ask questions such as: "How do the limbs and hooves of equines develop?" "How do the hooves work in locomotion?" "Why are the hooves singletoed?" Within the traditional framework of the proximate-ultimate distinction, the first two of these questions require hypotheses that invoke proximate causes, such as the biomechanics of how equine limbs and hooves work or the processes by which adult limbs and hooves arise during maturation. In the framework of the proximate-ultimate distinction, causes of this type (and the questions they are invoked to answer) are understood to be the proper focus of research in fields such as developmental biology, physiology, and anatomy.

In contrast, a concern with the latter question ("why are equine hooves single-toed?") would be a different endeavor - the study of ultimate causes. Ultimate causes "are responsible for the evolution of a particular DNA code of information with which every individual of every species is endowed" (Mayr 1961, p. 1503). They are population-level phenomena pertaining to evolution and history and account for why the species in question has evolved the trait of interest or exhibits a particular feature. Natural selection is the predominant ultimate cause, but there are other relevant candidates (e.g., drift or migration). For the equine single-toed hoof, an ultimate explanation might appeal to how ancestors of modern-day equines who had smaller second and fourth toes survived and reproduced at greater rates than those that did not (the last common ancestor of all equids lived approximately 4-4.5mya (Orlando et al. 2013)). This 
fitness difference resulted in diminishment and eventually a loss of function in these digits over evolutionary time.

It naturally follows from the proximate-ultimate distinction that the evolutionary sciences aim to discover and formulate explanations for phenomena in terms of ultimate causes. Although Mayr makes clear that reference to both types of causes is required for a full explanation of any given trait (i.e., the two causal domains are complementary), they are considered to be largely causally autonomous. Although knowledge of one causal domain can inform the other, the causal processes involved in each are distinct and require separate domains of inquiry; functional biologists (e.g., developmental biology or physiology) study proximate causes, and evolutionary biologists (e.g., population geneticists and behavioral ecologists) study ultimate causes.

More than 50 years after its initial articulation, Mayr's distinction has become prevalent within biology and evolutionary biology especially. This makes it difficult to discern that, rather than reflecting a deep causal chasm between the research domains of evolutionary biology and "proximate" sciences, the distinction reflects an underlying empirical and epistemic claim regarding the explanatory value of different kinds of explanations. Despite the appearance of some minimal causal relevance, it is largely assumed by many biologists that the mechanisms of trait development and physiology play no interesting explanatory role in the study of evolution and that the population-level dynamics of selection and drift play no explanatory role in the study of development and physiology. One can offer adequate answers to "how" questions without referring to ultimate causes and adequate answers to "why" questions without referring to proximate causes. However implausible this might seem, it is not difficult to see that the distinction can serve as a reasonable and useful idealization, such as to ignore or "black box" developmental mechanisms while undertaking evolutionary biological investigation. The assumption regarding the causal autonomy of proximate and ultimate domains is commonly invoked in evolutionary sciences but is perhaps best reflected within population genetics where little to no attention is paid to the specific mechanisms of variation and heredity within species. Within many genetic and molecular models of evolution, development is idealized away entirely, and the genotype-phenotype relationship is characterized purely mathematically with no reference to mechanism (Laubichler 2010).

\section{Motivating the Black-Boxing of Development}

Mayr's dichotomy reflects a mid-twentieth-century consensus within evolutionary biology, sometimes referred to as the Modern Synthesis, regarding the fundamental mechanics of evolution and the explanatory adequacy of population genetics. These ideas are still influential within evolutionary biology today and go a long way to explaining the continued appeal of the proximate-ultimate distinction. However, the exact nature of these empirical and theoretical commitments is complex, and there is not a single perspective that unites all evolutionary biologists apart from a basic commitment to the reality of evolution by natural selection as a phenomenon. Yet there are some key points upon which evolutionary biologists generally do agree and help illuminate how evo-devo and standard evolutionary theory converge and diverge.

First, both the Modern Synthesis and standard evolutionary theory are typically adaptationist in nature, although the contours of that commitment have changed over time (see the chapter on "Form and Function in Evo-Devo"). Historically, the adaptationism of evolutionary biology could be best summarized as the view that "natural selection is the predominant force in evolution." Sustained discussion in the wake of Gould and Lewontin's famous Spandrels 
critique ( 1979), coupled with theoretical and empirical advances, such as neutral theory (Kimura 1983), has led to a far more modest "explanatory" adaptationism in current evolutionary biology. Much contemporary evolutionary research is best characterized as motivated by the view that adaptation is the central evolutionary question and that evolution by natural selection is the primary, though not the only, explanation (Godfrey-Smith 2001; Orzack and Sober 1994).

Second, standard evolutionary theory primarily presents a gene-centric picture of evolution. It assumes that the biological structures that enable natural selection to operate are predominantly genetic and that mapping gene frequencies in populations over time is sufficient to capture the evolutionary process (Dobzhansky 1957, 1971; Mayr 1954; Mayr and Provine 1981). Although advances such as neutral theory have undermined the extent to which gene frequency change over time reflects selection alone, agreement remains among many evolutionary biologists that the evolutionary "action" occurs at a genetic level.

The appeal of this picture of natural selection and the explanatory adequacy of population genetics to many evolutionary biologists derive from two empirically motivated idealizations or simplifying assumptions about the relationship between genotypes and phenotypes (see the chapter on the "Genotype-Phenotype Map"). First, many evolutionary models assume that there is a smooth mapping from genotype to phenotype (i.e., small genetic changes map onto small phenotypic changes). Without such a strong correspondence, changes in phenotypes over time would not be accounted for solely by changes in genotypes, and therefore the explanatory adequacy of the gene-centric picture would be limited. Although modern evolutionary biologists are aware that the mapping is not always simple in nature due to factors such as neutrality, pleiotropy, and linkage effects (see the chapter on "Pleiotropy and Its Evolution: Connecting Evo-Devo and Population Genetics"), most maintain that macroevolution is merely the summation of microevolution over time. Mutation, selection, migration, and drift are sufficient to explain why populations exhibit specific traits with particular features across the tree of life.

A second common simplifying assumption is that the supply of phenotypic variation available to selection is isotropic over evolutionary timescales (i.e., the products of the developmental processes generating variation are roughly equiprobable in their occurrence). On this view, the mechanisms that produce phenotypic variation are not constrained or sensitive to the adaptive value of the variants that they generate. Again, although there is widespread acceptance that some phenotypic variants are more or less likely in the short term due to linkage effects and other molecular phenomena, the consensus is that these shorter-term effects will be washed out over evolutionary time. The isotropism assumption entails that the final outcomes of selective processes in populations are relatively causally autonomous from the processes responsible for phenotypic variation and development in organisms. The effectiveness of natural selection is dependent upon the presence of phenotypic variation within populations, but if that variation is assumed to be blind with respect to adaptation and more broadly unconstrained, then population-level processes account for the outcomes of the evolutionary process. This warrants black-boxing individual-level processes, such as developmental plasticity, which are believed to be of little to no explanatory importance. Despite the challenges this assumption faces due to recent empirical findings in the study of phenotypic plasticity and developmental constraint (West-Eberhard 2003), those working within the framework of standard evolutionary theory assume that these processes only play an important explanatory role in a small number of cases. Even when they are identified as playing a role, it is common to argue that their contribution 
nonetheless results from changes in gene frequency change over time (Wray et al. 2014; Welch 2017).

Both of these simplifying idealizations, and the associated broader assumptions about the process of evolution, are best understood as principled empirical "bets." No evolutionary biologist believes them to be exceptionless generalizations - it is implausible that there are law-like generalizations in biology (Beatty 1995). Rather, they are claims about what is taken to hold in the majority of circumstances. Given this, it is unsurprising that the proximateultimate distinction and the entailed understanding of the causal autonomy of evolution from development are generally accepted among biologists. Importantly, the rejection of these principled empirical "bets" motivates evo-devo and therefore involves a direct challenge of the dichotomy between proximate and ultimate causation (Laland et al. 2011).

\section{Evo-Devo: A Different Perspective}

Evo-devo is the current iteration of a much older historical movement that challenged the Modern Synthesis with its emphasis on the autonomy of the mechanisms of inheritance and variation from evolution (Laubichler 2010; Laubichler and Maienschein 2003; Love 2015a, b). This movement is motivated by a variety of empirical phenomena including phenotypic novelty, the persistence of homology, and genetic assimilation (see the chapters on " Developmental Homology," "Developmental Innovation and Phenotypic Novelty," and " Developmental Plasticity and Evolution"). Those within evo-devo argue that these phenomena fail to fit within the picture of evolution presented by the Modern Synthesis, and more specifically, they contradict the empirical simplifying assumptions regarding the genotypephenotype map and the nature of the supply of phenotypic variation to selection.

Consider homologies - traits that are shared between species in virtue of sharing a common ancestor. According to standard evolutionary theory, these are simply "ancestral characters that happened by circumstance to survive" (Williams 1992, p. 99). They are the reflection of a shared genetic inheritance through common ancestry and eventually will be "washed out" of the genotype through the steady appearance of random mutations and selection (Amundson 2005). This view is, however, contradicted by evidence that many homologies are the product of more than just residual genetic inheritance. There are conserved, shared developmental mechanisms that are both causally responsible for homologies and appear to facilitate their persistence over evolutionary time by protecting the phenotype from environmental and genetic perturbation (Wagner 2014; see also the chapters on "Developmental Homology" and "Typology and Natural Kinds in Evo-Devo"). This understanding of the mechanisms of homology challenges the notion that homologous traits would be "washed out" by mutation and selection; it also undermines the isotropism assumptions about variation in standard evolutionary theory. Although the supply of mutations within populations may be isotropic, the supply of phenotypic variation to selection is not. The developmental mechanisms underwriting many homologues serve to make particular phenotypic possibilities more likely than others (see the chapter on "Dispositional Properties in Evo-Devo"). Moreover, homologies are ubiquitous across the tree of life and not exceptional cases. Evolutionary developmental biologists take this type of evidence to undermine the justification for a principled empirical bet in favor of an isotropic supply of phenotypic variation to selection and motivate the study of the relationship between the developmental mechanisms underwriting homologies and evolution more generally.

Phenomena like this have led evo-devo biologists to emphasize, rather than downplay, the complexity of the relationship between genes and phenotypes and reject the proximate-ultimate 
distinction. Rather than adopting the empirical bets of standard evolutionary theory, evo-devo works from a different perspective, one that focuses on the construction of the phenotype and the ways in which the developmental processes responsible for the phenotype can influence evolution.

Given this alternative perspective, it is unsurprising that evo-devo proponents often directly challenge the proximate-ultimate distinction. Although there is some controversy regarding the exact nature of the challenge (Minelli 2010; Laubichler 2010; Laland et al. 2011, 2014; Wray et al. 2014; Welch 2017), there are two important commitments in evo-devo as a research program that contradicts Mayr's account of biological causation.

The most obvious and salient of these commitments is the important role that evo-devo biologists see development playing in evolution (Laland et al. 2011, 2014). According to the traditional proximate-ultimate distinction, the mechanistic, individual-level causal processes of development are assumed to be relatively independent of the population-level processes of evolution by natural selection. Evo-devo biologists clearly reject this assumption. Developmental processes are central to understanding a number of important evolutionary "why" questions, such as the origin of phenotypic novelties (innovation), trait identity in the tree of life (homology), and the distribution of phenotypes across that tree (disparity and evolvability). Returning to the single-toed equine hoof, an adequate explanation of why horses have the hooves they do - from an evo-devo perspective - involves reference not just to selection but also to the developmental mechanisms that made the diminishment of the second and fourth digits possible (Alberch and Gale 1985). It requires understanding how the supply of phenotypic variation required for the evolution of the single-toed hoof arose. Appealing to the simple accumulation of mutation is inadequate. In this sense, evo-devo biologists deny the strong causal autonomy entailed by the proximate-ultimate distinction: answering evolutionary questions requires reference to development.

The second evo-devo commitment challenges the proximate-ultimate distinction more directly. Evo-devo biologists engage in a particular type of explanation that sits entirely outside of the dichotomous picture Mayr's distinction presents (Calcott 2013; Laubichler 2010). Evo-devo researchers emphasize the complexity that the construction of the organism presents to evolutionary processes. The question of how one evolves a limb or another bodily trait cannot be answered by simple reference to mutation and selection (Kirschner and Gerhart 2005). Unsurprisingly, explanations in evo-devo often consist in offering a step-by-step account of how a trait evolved via small changes to the mechanisms underwriting it. In the case of the equine single-toed hoof, the stepwise progression of cumulative developmental changes required to get from a three-toed to single-toed hoof would comprise such an explanation. These so-called lineage explanations (Calcott 2009) fall outside of the "how" and "why" dichotomy of the proximate-ultimate distinction. They are neither mechanistic answers to "how" questions or historic population-level answers to "why" questions. They rather explain the evolution of a trait by reference to changes in the mechanisms that are responsible for it without reference to selection or populations.

The picture of causation and explanation that this leaves us with differs dramatically from that originally presented by Mayr. Mayr emphasized a dichotomy between individual-level causes, for which we give mechanistic explanations, and population-level causes, for which we give historical explanations. In contrast, evo-devo biologists demonstrate the relevance of individual-level causes and mechanistic explanations to evolutionary questions. Historical explanations, contra Mayr, can be both mechanistic and individual level in nature. Although 
further empirical work is required to cash out the empirical bets of evo-devo, new techniques and advances within developmental genetics and embryology now make it possible to more rigorously test this picture of evolution and thereby its associated account of evolutionary inquiry.

\section{Cited Works}

Alberch P, Gale EA (1985) A developmental analysis of an evolutionary trend: digital reduction in amphibians. Evolution 39:8-23

Amundson R (2005) The changing role of the embryo in evolutionary thought: the roots of evo-devo. Cambridge

CrossRef University Press, Cambridge

Beatty J (1994) The proximate/ultimate distinction in the multiple careers of Ernst Mayr. Biol Philos 9(3):333356

Beatty J (1995) The evolutionary contingency thesis. In: Wolters G, Lennox JG (eds) Concepts theories, and rationality in the biological sciences. University of Pittsburgh Press, Pittsburgh

Calcott B (2009) Lineage explanations: explaining how biological mechanisms change. Br J Philos Sci 60(1):5178

Calcott B (2013) Why how and why aren't enough: more problems with Mayr's proximate-ultimate distinction. Biol Philos 28(5):767-780

Dobzhansky TG (1957) On methods of evolutionary biology and anthropology. Am Sci 445:381-392

Dobzhansky TG (1971) Genetics of the evolutionary process. Columbia University Press, New York

Godfrey-Smith P (2001) Three kinds of adaptationism. In: Orzack SH, Sober E (eds) Adaptationism and optimality. Cambridge University Press, Cambridge, pp 335-357

Gould SJ, Lewontin RC (1979) The spandrels of San Marco and the Panglossian paradigm: a critique of the adaptationist programme. Proc R Soc Lond B 205:581-598

Kimura M (1983) The neutral theory of molecular evolution. Cambridge University Press, Cambridge

Kirschner MW, Gerhart JC (2005) The plausibility of life: resolving Darwin's dilemma. Yale University Press, New Haven/London

Laland KN, Sterelny K, Odling-Smee J, Hoppitt W, Uller T (2011) Cause and effect in biology revisited: is Mayr's proximate-ultimate dichotomy still useful? Science 334:1512-1516

Laland KN, Uller T, Feldman M, Sterelny K, Müller GB, Moczek A, Jablonka E, Odling-Smee J (2014) Does evolutionary biology need a rethink? Point: yes, urgently. Nature 514:161-164

Laubichler MD (2010) Evolutionary developmental biology offers a significant challenge to the neo-Darwinian paradigm. In: Ayala FJ, Arp R (eds) Contemporary debates in philosophy of biology. Wiley-Blackwell, Malden, pp 199-212

Laubichler M, Maienschein J (eds) (2003) From embryology to Evo-Devo: a history of developmental evolution. MIT Press, Cambridge, MA

Love AC (2015a) Conceptual change in evolutionary developmental biology. In: Love AC (ed) Conceptual change in biology: scientific and philosophical perspectives on evolution and development. Boston studies in philosophy of science. Springer, Berlin, pp 1-54

Love AC (2015b) Evolutionary developmental biology: philosophical issues. In: Heams T, Huneman P, Lecointre G, Silberstein M (eds) Handbook of evolutionary thinking in the sciences. Springer, Berlin, pp 265-283

Mayr E (1954) Change of genetic environment and evolution. Evol Process 157:180

Mayr E (1961) Cause and effect in biology. Science 134:1501-1506

Mayr E, Provine WB (1981) The evolutionary synthesis. Bull Ame Acad Arts Sci 17-32

Minelli A (2010) Evolutionary developmental biology does not offer a significant challenge to the neo-Darwinian paradigm. In: Ayala FJ, Arp R (eds) Contemporary debates in philosophy of biology. Wiley-Blackwell, Malden, pp 213-226

Orlando L et al (2013) Recalibrating Equus evolution using the genome sequence of an early middle Pleistocene horse. Nature 499:74-78

Orzack SH, Sober E (1994) Optimality models and the test of adaptationism. Am Nat 143(3):361-380

Wagner G (2014) Homology, genes, and evolutionary innovation. Princeton University Press, Princeton

Welch JJ (2017) What's wrong with evolutionary biology? Biol Philos 32:273-279

West-Eberhard MJ (2003) Developmental plasticity and evolution. Oxford University Press, New York

Williams GC (1992) Natural selection: domains, levels and challenges. Oxford University Press, Oxford

Wray GA, Futuyma DA, Lenski RE, MacKay TFC, Schluter D, Strassman JE, Hoekstra HE (2014) Does evolutionary biology need a rethink? Counterpoint: no, all is well. Nature 514:161-164 